\title{
Das epistemische Verhältnis der Sozialrobotik zur Gesellschaft
}

\section{Epistemische Bedingungen, Praktiken und Implikationen der Entwicklung sozialer Roboter}

Andreas Bischof

\begin{abstract}
Of course, the robots that now exist and that are described in fascinating detail in this book that you are holding, are not yet as complex, versatile and intelligent as the imaginary robots of I, Robot, but give the engineers time! There will be steady advances in robotics, and, as in my teenage imagination, robots will shoulder more and more of the drudgery of the world's work, so that human beings can have more and more time to take care of its creative and joyous aspects.
\end{abstract}

(Isaac Asimov in Robotics in Practice von Joseph Engelberger)

\section{Zusammenfassung}

Soziale Roboter kommen schon sozial und kulturell geprägt in die Welt. Das Kapitel nähert sich diesem Befund über die Analyse der epistemischen Praktiken der Sozialrobotik. Dabei werden Wissenspraktiken aus den Disziplinen, die an der Konzeption und Konstruktion von sozialen Robotern beteiligt sind, analysiert. In einem ersten Schritt umfasst das die Darstellung von epistemischen Bedingungen, unter denen die Konstruktion sozialer Roboter überhaupt geschieht. Hier wird insbesondere auf die Herausforderung, soziale Interaktionen für Roboter bearbeitbar zu machen, eingegangen. In einem zweiten Schritt werden typische epistemische Praktiken der Sozialrobotik rekonstruiert. Damit sind Tätigkeiten der Forschenden gemeint, die soziale Roboter besser für soziale Welten verfügbar machen - oder umgekehrt. Sie beschreiben Wechsel-

\footnotetext{
A. Bischof $(\bowtie)$

TU Chemnitz, Chemnitz, Deutschland

E-Mail: andreas.bischof@informatik.tu-chemnitz.de
}

(C) Der/die Autor(en) 2021

O. Bendel (Hrsg.), Soziale Roboter, https://doi.org/10.1007/978-3-658-31114-8_2 
spiele zwischen der Forschungs- und Entwicklungspraxis und den Alltagswelten, in denen die Roboter eingesetzt werden sollen. Abschließend werden zwei epistemische Implikationen für die Anwendungsfelder sozialer Roboter abgeleitet.

\subsection{Einleitung}

Unter dem Begriff „epistemisch“ (engl. epistemic) wird in der Wissenschafts- und Technikforschung untersucht, welchen erkenntnistheoretischen Grundlagen ein wissenschaftliches Feld oder anderer sozialer Zusammenhang unterliegt. Dabei interessieren ganz grundlegend die Wege und Mittel, mit denen Wissen erzeugt, zirkuliert und legitimiert wird - wie wissen wir, was wir wissen?

Die Arbeit an sozialen Robotern steht in einem sehr spezifischen Verhältnis zur Gesellschaft. Der Schriftsteller Isaac Asimov, der das Wort „Robotik“ in einem seiner Werke 1942 erstmals verwendete (Clarke 2011, S. 256), setzt es in oben stehendem Zitat in ein sehr eindeutiges Bild: Die Imaginationskraft des Schriftstellers ist der Auftrag der Ingenieure, die einzig mehr Zeit bräuchten, um entsprechend komplexe, vielseitige und intelligente Roboter zu bauen, wie die Fantasie sie hervorzubringen vermag. Der Weg zu ihrer technischen Realisierung sei durch stetigen Fortschritt gekennzeichnet. Dass ein Autor fiktionaler Literatur dieses Versprechen im Vorwort zu einem wissenschaftlichen Handbuch aus der Feder eines Robotikpioniers macht, gibt einen Ausblick auf die komplizierten epistemischen Wechselwirkungen, unter denen soziale Roboter entwickelt werden. Und diese Wechselwirkungen zwischen Robotik und sozialen Welten sind mitnichten so einseitig, wie Asimov sie beschrieb - mittlerweile betätigen sich sogar Robotiker als Science-Fiction-Autoren (Nourbakhsh 2013)!

Die Konzeption, der Bau und die Evaluation sozialer Roboter sind Gegenstand der Disziplin Sozialrobotik bzw. des interdisziplinären Forschungsfelds Human-Robot Interaction (HRI). Im Vergleich zu anderen Forschungsfeldern, wie z. B. Philosophie, aber auch Human-Computer Interaction, wird die Frage nach der Epistemologie innerhalb der Sozialrobotik selten explizit gestellt. ${ }^{1}$ Allerdings existieren eine Reihe sozial- und kulturwissenschaftlicher Studien mit Beiträgen zur Epistemologie der (Sozial-)Robotik und Mensch-Roboter-Interaktion (Šabanović 2007; Meister 2011; Treusch 2015; Bischof 2017; Lipp 2019). Dieses Kapitel gibt einen Überblick über die wesentlichen Bedingungen, Praktiken und Implikationen der epistemischen Grundlagen der Sozialrobotik. In der Untersuchung der Wissensweisen der Sozialrobotik behandelt das Kapitel also nicht die philosophischen Grundlagen der Möglichkeit sozialer Maschinen - sondern vielmehr die

\footnotetext{
${ }^{1}$ Eine Volltextsuche unter den Vortragstiteln auf den zentralen Konferenzen HRI (ACM), RO-MAN (IEEE) und Social Robotics (Springer) zwischen 2012 und 2021 ergibt sieben Treffer, wobei kein Beitrag davon die epistemischen Grundlagen der Sozialrobotik selbst thematisiert, sondern „epistemisch“ bspw. für die Kognition von Menschen verwendet wird.
} 
oftmals unsichtbaren Bedingungen und Praktiken, mittels derer soziale Roboter überhaupt in die Welt kommen. Dieser praxeologische Zugang steht in der Tradition der ,Laborstudien“ (Krey 2014), die die Wissenschafts- und Technikforschung seit Ende der 1970erJahre dorthin führen, wo Wissen wortwörtlich gemacht wird, in den Alltag der Forscherinnen und Forscher.

Damit ist erstens eine wesentliche epistemische Entscheidung getroffen, die zu Beginn kurz erläutert werden muss. Handlungsleitend für das Vorgehen, die Wissensweisen der Sozialrobotik empirisch aus alltäglichen Praktiken heraus zu erforschen, ist die These, dass alles Wissen - auch wissenschaftliche Erkenntnis - sozial geformt ist. Das bedeutet nicht, dass die unserer Existenz zugrunde liegenden physikalischen Gesetze sozial konstruiert sind! Sehr wohl aber sind ihre Entdeckung und Folgen gesellschaftlich geprägt. Ein drastisches Beispiel dafür liefert die kopernikanische Wende: Die Erde hat sich bereits um die Sonne gedreht, als der Großteil der Menschheit noch an andere Himmelsvorstellungen geglaubt hat. Gleichsam hatte die Durchsetzung der Erkenntnis, dass die Erde mitnichten das Zentrum des Weltalls (nicht einmal das Zentrum unseres Sonnensystems) ist, auch soziale Folgen: Das heliozentrische Weltbild hat nicht nur astronomische Theorien und wissenschaftliche Praxis verändert, sondern auch das allgemeine Weltbild (Kuhn 1957).

Für die Beschreibung und Erklärung der Epistemologie eines Forschungsfelds ist zweitens zu untersuchen, wie wissenschaftliches Wissen praktisch hervorgebracht wird. Für die von den frühen Laborstudien erforschten Naturwissenschaften sind die namensgebenden Labore als Orte der „Erzeugungslogik“ moderner Wissenschaften zentral (Knorr Cetina 1988, S. 88): Dort werden die Gegenstände der Untersuchung, seien es biochemische Moleküle, hochenergetische Teilchen oder Himmelskörper, räumlich und zeitlich entkoppelt und der wissenschaftlichen Untersuchung zugänglich gemacht. Im Labor lassen sich Forschungsgegenstände, die Instrumente und Praktiken ihrer Behandlung und die Herstellung wissenschaftlicher Fakten im Zusammenspiel beobachten. Für soziale Roboter liegt der Fall etwas komplizierter, wie im Fortgang zu zeigen sein wird: Denn das Labor der Sozialrobotik sind nicht nur die „Labs“, in denen die Robotikerinnen und Robotiker ihre Maschinen bauen und testen. Die Epistemologie der Sozialrobotik formt sich auch auf den Konferenzen, auf denen die Maschinen einander vorgeführt werden, durch YouTube-Videos von Roboterverhalten und nicht zuletzt durch die sozialen Welten, an denen die Robotikerinnen und Robotiker selbst als Alltagsmenschen teilnehmen.

\subsection{Epistemische Bedingungen der Sozialrobotik}

Soziale Roboter zu bauen, ist eine unerhörte Herausforderung. Diese Herausforderung besteht einerseits in der Komplexität der Aufgabe, überhaupt eine funktionierende Maschine zu bauen. Schon ein vergleichsweise einfacher Roboter besteht aus zahlreichen, an sich komplizierten Komponenten, die zudem miteinander in Zusammenhang gebracht werden müssen, um schließlich in einer Umgebung, die für Menschen statt Roboter gemacht ist, zu funktionieren. Andererseits sind soziale Roboter eine Aufgabe, die die klas- 
sischen Mittel von Ingenieur- und Naturwissenschaften übersteigt. Im Folgenden wird gezeigt, worin diese unerhörte Herausforderung besteht, und wie sie wiederum selbst gesellschaftlich eingebettet ist.

\subsubsection{Mensch-Maschine-Symbiose als „Wicked Problem“}

Dass soziale Roboter überhaupt zu einem Ziel der Robotik werden konnten, geht auf einen Paradigmenwechsel innerhalb der Robotik und K.I.-Forschung in den 1980er-Jahren zurück. Dieser Paradigmenwechsel basiert auf einer Verschiebung des Verständnisses von maschineller Intelligenz hin zu natürlichen Vorbildern statt symbolisch-kognitivistischen Konzepten. Einer der Protagonisten dieser „New Wave of Robotics“ war Rodney A. Brooks. Anhand des von ihm begründeten Ansatzes der ,behavior-based robotics“ "lässt sich diese neue konzeptionelle Qualität im Verhältnis von Roboter und Welt anschaulich zusammenfassen (Brooks 1999, S. 64 ff.).

Erstens stellten die Robotikforschenden fest, dass Alltagsmenschen ganz andere Lösungsstrategien als das in der K.I. bis dato modellierte ,problem solving“ verwenden, nämlich routinisierte Handlungsabläufe anstatt formallogischer Vorgehensweisen. Zweitens erkannte man, dass Menschen anderen Menschen - aber auch Tieren und sogar Objekten - Ziele und Vorstellungen unterstellen, unabhängig davon, ob diese tatsächlich höhere symbolische Repräsentationen prozessieren können. Vielmehr sind Menschen sehr leicht geneigt, allein durch das Zeigen von (scheinbar) autonomem Verhalten dem Gegenüber Intentionalität zuzuschreiben. Brooks selbst hatte, biologischen Vorbildern folgend, drittens nachgewiesen, dass Roboter keine vollständige, hierarchische Softwarearchitektur benötigen, um kompetent in einer Umwelt agieren zu können. An die Stelle einer zentralen kognitivistischen Steuerung trat eine niedrigschwellige Koordinierung einzelner Systeme, die mit der Welt interagieren, wie zum Beispiel Sensoren und Aktuatoren, die einfachen, regelgeleiteten Prinzipien folgend Dinge in der Welt wortwörtlich begreifen.

Mit der „New Wave of Robotics“ setzt sich in der Robotik die Vorstellung durch, dass die Entwicklung erfolgreicher Roboter an ihren Einsatzumgebungen, der „realen Welt“, orientiert sein muss. Damit wird die Performance der Maschinen in unstrukturierten Umgebungen zum Hauptkriterium ihrer Bewertung. Während die Protagonisten der „New Wave of Robotics“ vor allem das Problem einer physikalisch und biologisch adäquaten Interaktion mit Objekten und Räumen lösen wollten, ist das Prinzip der Orientierung an der ,realen Welt“ für soziale Roboter umso folgenreicher: Für diese stellen sich nicht nur physische Bedingungen für gute Performance, sondern psychologische und soziale. Sozialität ist allerdings wechselseitig - und geht somit über das technische System hinaus. Die wesentliche Gelingensbedingung, sich in der Welt zu bewähren, kann von Sozialrobotern also nicht allein erfüllt werden, sondern nur im erfolgreichen Zusammenspiel mit Menschen. Meister hat gezeigt, wie fundamental diese Bedingung ist:

Die Problematik einer möglichst reibungsfreien und intuitiven Nutzbarkeit wird nun keineswegs nur als eine technische Aufgabenstellung diskutiert, sondern als ein Bestandteil des 
Verständnisses von intelligenten Systemen überhaupt: Diese Roboter sollen „Kooperationsfähigkeit“ $[\ldots]$ besitzen, und letztlich wird als Trägerin der Intelligenz nicht das isolierte technische System, sondern die schon genannte „Mensch-Maschine-Symbiose“ verstanden. (Meister 2011, S. 108)

Soziale Welten sind für Roboter nicht nur physisch anspruchsvoll. Soziale Interaktionen sind abhängig von Interpretationen, die nicht zwangsläufig deduktiv vorhersagbar sind und sich zudem durch die Einnahme unterschiedlicher Beobachterpositionen verändern (Lindemann 2016). Es darf nicht unterschätzt werden, was diese Verschiebung des Fokus auf das Gelingen in Interaktion mit Menschen bedeutet. Diese Aufgabe liegt nicht nur quer $\mathrm{zu}$ vorherigen Interessen und Arbeitsweisen in der Robotik als technikwissenschaftlicher Disziplin, sie erschüttert auch ihre erkenntnistheoretischen Grundlagen. Durch den angestrebten Einsatz in alltäglichen Lebenswelten wird Sozialrobotik plötzlich zu einer Disziplin wie Architektur oder Stadtplanung, in der sich wissenschaftliche, technische, politische, soziale und ästhetische Expertisen und Interessen kreuzen. Das liegt daran, dass Sozialrobotik und Architektur nun die gleiche Art von Problem teilen: das der widerständigen - einige sagen bösartigen (Rittel und Webber 1973) - Natur der (Un-)Vorhersagbarkeit menschlichen Verhaltens in soziotechnischen Systemen. Das heißt, dass die zu lösenden Probleme der Sozialrobotik so komplex und veränderlich sind, dass keine standardisierten Lösungen bestehen. Stattdessen hängt die Bearbeitung von ,wicked problems" wesentlich von der Formulierung und Definition des Problems durch die Entwickelnden selbst ab (ebd.). Die Lösung eines ,wicked problem“ ist deshalb ein Prozess, der neue Wissenssorten, Fähigkeiten und Perspektiven nötig macht, die zuvor nicht zum Selbstbild oder zur Ausbildung der Robotik gehörten.

Wie die Robotikforschung seit dem Verlassen der Labore auf unterschiedlichen Wegen erlebt, funktionieren auch scheinbar triviale Ausschnitte von Alltagswelt wie Krankenhausflure als Lebenswelten in ihrem jeweils eigenen Sinn (Mutlu und Forlizzi 2008). Die spezifische Komplexität der Alltagswelt ist deswegen die Kernherausforderung der Sozialrobotik (Meister 2014; Bischof und Maibaum 2020). Die Entwicklerinnen und Entwickler von sozialen Robotern müssen deswegen stetig work arounds erarbeiten, um einen Begriff aus dem Programmierslang zu verwenden: Sie entwickeln Hilfsverfahren, die das eigentliche Problem (die rechentechnisch schwer darstellbare Komplexität alltagsweltlicher Umgebungen) nicht lösen, sondern dessen Symptome umgehen, um zumindest die ersten Interaktionshürden zu nehmen. Dieses Zum-Funktionieren-Bringen von Robotern in sozialen Welten ist eine integrative und komplexe Praxis (Nourbakhsh, nach Šabanović 2007, S. 204), die über das Lösen technischer Probleme weit hinausgeht.

\subsubsection{Soziale Roboter als Realisierung von Zukunftsvorstellungen}

Bevor die Praxis der Sozialroboterentwicklung näher betrachtet wird, ist eine zweite zentrale epistemische Bedingung für soziale Roboter darzustellen: Schon die Idee zu sozialen Robotern ist sozial und kulturell geformt. Zwar lassen sich diese mit wissenschaftlichen 
Methoden werturteilsfrei beschreiben und untersuchen, aber die Sozialrobotik selbst ist stark gesellschaftlich geprägt: Erstens birgt die Idee für Maschinen, die menschliche Tätigkeiten verrichten, ein großes und nicht widerspruchsfreies kulturelles Erbe, und zweitens findet auch die konkrete Finanzierung der Entwicklung von sozialen Robotern nicht ohne gesellschaftlichen Auftrag statt.

Beide Dimensionen sind zudem miteinander verschränkt, wie die Untersuchung von Weltbildern und Zukunftsvorstellungen in der Sozialrobotik zeigt (Šabanović 2010; Böhle und Bopp 2014; Lipp 2019). Dabei kann gezeigt werden, dass die finanzierenden Institutionen, die Konstrukteurinnen und Konstrukteure, aber auch die später mit den Roboter interagierenden Menschen sich in einem Zusammenspiel aus technischen Möglichkeiten, dem Wunsch danach und kollektiven Vorstellungen beeinflussen (Jasanoff und Kim 2009, S. 120). Zentral ist dabei die Erkenntnis, dass auch die Vorstellung einer einzelnen MenschRoboter-Interaktion, wie bspw. eine Roboterunterstützung für Menschen mit Demenz, fast zwangsläufig in umfassendere Visionen von sozialer Zukunft, von Risiko und Nutzen und vom individuellen wie kollektiven Wohl eingebettet ist. Mit dem Begriff ,imaginary“ (ebd.) wird in der Wissenschafts- und Technikforschung diese Figur der wechselseitigen Ko-Konstitution sozialer und technischer Zukunftsvorstellungen beschrieben. ${ }^{2}$ In der Sozialrobotik treten das technisch Machbare und das gesellschaftlich Wünschenswerte in besonders enger Kopplung auf. Das lässt sich für den europäischen Raum am Beispiel der Pflegerobotik (Bendel 2018; Hergesell et al. 2020) und ihrer Forschungsförderung gut zeigen: Als Antwort auf ein erwartetes Problem wird eine politisch gewünschte Zukunftsvorstellung als technisch erreichbar verstanden, womit anschließend Investitionen und entsprechende Förderlinien begründet werden (Lipp 2019, 2020). Empirische Untersuchungen von Pflegerobotikprojekten zeigen, dass dieses Ziel in der Praxis dann immer wieder zur wechselseitigen Anpassung der Roboter an die Pflege und umgekehrt herangezogen wird - wobei die ursprüngliche Problem-Lösung-Relation nicht mehr hinterfragt werden kann (Bischof 2020; Maibaum et al. 2021).

In Rückgriff auf Suchman (2007) nutzt Šabanović ,,imaginaries“ als zentrale Analyseeinheit für ihre umfangreiche ethnografische Studie zur Kultur der Sozialrobotik (Šabanović 2007). Der Reiz liegt dabei für sie darin, dass ,,imaginaries“ auch als kulturelle und historische Ressourcen verstanden werden können (ebd., S. 29). Auch technisch derzeit noch nicht erreichbare Zukunftsvorstellungen von Robotern in der Gesellschaft können zum wechselseitigen Bezugspunkt zwischen Robotikforschenden und gesellschaftlichen Gruppen werden (ebd., S. 45). Ein augenfälliges Beispiel dafür sind die zahlreichen Bezüge zu Science-Fiction-Filmen, die in Presseberichten über soziale Roboter, aber auch durch die Forschenden selbst hergestellt werden (Meinecke und Voss 2018; Voss 2021).

\footnotetext{
${ }^{2}$ Das ,„socio-technical imaginary“ unterscheidet sich von anderen Begriffsangeboten wie dem „Diskurs“, der hauptsächlich auf Sprache fokussiert. „Ideologie“ wäre wiederum mehr auf Macht und soziale Struktur abgestimmt und damit statischer. Über „Interesse“ oder „Plan“ geht es insofern hinaus, als es sich nicht (nur) auf kurzfristige Zukünfte mit spezifischen Zielen (z. B. Plan zum Bau einer Autobahn) bezieht und nicht an einzelne Handelnde gebunden ist.
} 
Überhaupt ist es erwähnenswert, dass sowohl der Begriff „Roboter“ (Čapek 2014) als auch „Robotik“ (Asimov 2014) literarische Erfindungen sind, auf die sich schon die frühen Pioniere der Robotik wiederum explizit bezogen haben: Roboter waren also fiktionale Apparate, lange bevor sie funktionierende Maschinen wurden (Bischof 2017, S. 138-145). Untersuchungen von ,imaginaries“ in der Sozialrobotik zeigen dementsprechend, dass neben wissenschaftlichen Theorien und Methoden beim Entwickeln sozialer Roboter auch kulturelle Ressourcen wie Geschichten und Symbole eine wichtige Rolle spielen. Prominente soziale Roboter werden sogar selbst zu solchen Symbolen, wenn sie bspw. zu öffentlichen Anlässen als Botschafter eingesetzt werden. Das prominenteste Beispiel dafür derzeit ist der menschenähnlich gestaltete Roboter „Sophia“ von Hanson Robotics, der 2017 öffentlichkeitswirksam die saudische Staatsbürgerschaft verliehen bekam - was in starkem Gegensatz zu den tatsächlichen Fähigkeiten des Roboters und dem Konzept der Staatsbürgerschaft steht (Parviainen und Coeckelbergh 2020).

Mit Blick auf historische technische und wissenschaftliche Leistungen wie die Mondlandung oder die Entdeckung und Nutzbarmachung der Kernspaltung kann es prinzipiell nicht überraschend sein, dass ein komplexes Unterfangen wie der Bau von sozialen Robotern wesentlich von sozialen und politischen Visionen und Wünschen angetrieben ist. Für die Sozialrobotik spezifisch ist die vergleichsweise sehr enge Verknüpfung von populären Roboterbildern und Zukunftsvorstellungen mit konkreter Forschungsförderung, Projektanträgen, öffentlichen Vorträgen und Studieninhalten innerhalb der Sozialrobotik - und wiederum den Erwartungen von Menschen, die potenziell mit den Robotern interagieren können sollen. Hier ist insbesondere für Japan und den südostasiatischen Raum eine große Wirksamkeit dieser ,imaginaries“ bis hin in die letztlich resultierende Mensch-RoboterInteraktion nachgewiesen (Wagner 2013). Im europäischen Raum wiederum ist, wie oben dargestellt, ein sehr starkes innovationspolitisches ,imaginary“ wirksam, das das Projekt von sozialen Robotern in der Altenpflege als notwendige und wünschenswerte Lösung für den bevorstehenden demografischen Wandel positioniert.

Soziale Roboter können nicht ohne diesen spezifischen kulturellen Hintergrund verstanden und bewertet werden. Außerdem sollten vorgeschlagene robotische Lösungen für soziale Probleme nicht als gegeben hingenommen werden, sondern als Produkt politischer, technologischer und sozialer Prozesse, die die Idee sozialer Roboter erst möglich machen.

\subsection{Epistemische Praktiken der Sozialrobotik}

Das „wicked problem“ der Sozialrobotik besteht im Umgang mit schwer standardisierbaren, mehrdeutigen Phänomenen wie sozialen Interaktionen, die auf verlässlich messbare und rechentechnisch abbildbare Werte gebracht werden müssen. Zudem zeichnet sich mit Blick auf die ,imaginaries“ ein Bild von Roboterentwicklung als ein in mannigfaltige kulturelle und soziale Instanzen eingelassenes Ensemble, das über vermeintlich ,rein “ technische oder wissenschaftliche Fragen weit hinausgeht. Die spannende Frage ist, wie 
die Entwicklerinnen und Entwickler mit diesen beiden zuvor dargestellten epistemischen Bedingungen umgehen.

Der empirische Schlüssel dafür sind die beobachtbaren lokalen Praktiken der Sozialrobotik, die gleichzeitig immer als durch materielle, institutionelle und symbolische Umstände vermittelt verstanden werden müssen: So ist die Entscheidung eines Post-Docs für die experimentell begründete Auswahl einer bestimmten Roboterbegrüßungsgeste nicht ohne dessen Wissen um hinreichend gute Signifikanzen quantitativer Daten für die Akzeptanz von Forschungspublikationen in seinem Feld verstehbar (Bischof 2017, S. 225 ff.). An diesem Beispiel zeigt sich der Vorteil einer praxeologischen Laborstudie: Durch sie lassen sich in der offiziellen Selbstdarstellung der Sozialrobotik unsichtbare Praktiken sichtbar machen - und auf ihre erkenntnistheoretischen Grundlagen befragen. Das ist insbesondere für Robotik wichtig, da sehr viele arbeitsteilige, komplizierte Arbeitsschritte zwischen dem Entwurf, der Fertigung und Evaluation eines Roboters nötig sind, die notwendigerweise nicht alle zu gleichen Teilen in einem Forschungsaufsatz oder Selbstzeugnis aus dem Feld der Robotik dargestellt werden können.

Im Folgenden werden drei auf diese Art erforschte Gruppen epistemischer Praktiken dargestellt, die für die Sozialrobotik typisch sind (Bischof 2017, S. 213-268). Mit dem Begriff ,epistemische Praktiken“ sind in diesem Zusammenhang Tätigkeiten gemeint, die mit dem dargestellten ,wicked problem“ produktiv umgehen, die Forscherinnen und Forscher also auf dem ein oder anderen Weg in die Lage versetzen, ihre Maschinen besser für soziale Welten verfügbar zu machen, oder umgekehrt. Sie beschreiben nicht die einzelnen Versuchsanordnungen der Laboratorien oder individuelle Vorstellungen von ,guter ${ }^{6}$ Mensch-Roboter-Interaktion innerhalb eines Forschungsteams, sondern die Wechselspiele zwischen der Forschungs- und Entwicklungspraxis und den Alltagswelten, in denen die Roboter eingesetzt werden sollen.

\subsubsection{Laboratisierende Praktiken}

Schon aus Sicherheitsgründen ist eine naheliegende Strategie, mit den Unwägbarkeiten von (Robotern in) sozialen Welten umzugehen, diese in die kontrollierten Bedingungen eines Labors zu überführen. Paradigmatisch steht dafür das Laborexperiment, bei dem unter Ausschluss von Umwelteinflüssen ein oder mehrere Faktoren gezielt auf ihre Effekte getestet werden können. Die Kontrollierbarkeit der Mensch-Roboter-Interaktion ist das zentrale Ziel. Der Erfolg dieser Strategie hat in der Sozialrobotik zur Vorherrschaft des quantitativen Laborexperiments als wichtigstem wissenschaftlichen Gütekriterium geführt (Bischof 2015). Die Fixierung auf diese psychologische Methode hat auch zu Identitäts- und Machtkonflikten im interdisziplinären Feld geführt, die heute noch auf Konferenzen oder in Gutachten für Artikel und Anträge zu beobachten sind. In die Worte eines eher ingenieurwissenschaftlich geprägten Forschers gefasst lautet er: „Bauen wir Roboter, um Experimente zu machen, oder machen wir Experimente, um Roboter zu bauen?" (ebd.; Bischof 2017, S. 214-216) 
Der Erfolg der Laborexperimente erscheint auch vor dem Hintergrund des eigentlichen Ziels, Roboter in realen Welten zum Funktionieren zu bringen, kontraintuitiv. Auf forschungspraktischer Ebene erklärt sich ihr Erfolg auch in der besseren Handhabbarkeit: Die Instrumente und Praktiken der Laboratisierung ermöglichen eine Beschreibung von Mensch-Roboter-Interaktion in quantitativen Werten, die (scheinbar) leichter rechentechnisch für die Steuerung der Maschinen weiterverwendbar sind. Das lässt sich am Beispiel der Operationalisierung von Emotionen mit der Methode FACS gut zeigen.

Emotionen haben als Faktor zur Gestaltung sozialer Roboter eine lange Tradition (z. B. Breazeal 2003). Einerseits wurde daran gearbeitet, Roboter durch vorgegebene oder selbst, wahrgenommene' Faktoren emotionale Zustände ausdrücken lassen zu können. Um das zu erreichen, wird aus einem Set von Grundemotionen, wie Freude oder Furcht, ein mehrdimensionaler Raum aufgespannt, in dem dann künstliche Gefühlszustände berechnet werden können (Becker-Asano und Wachsmuth 2008). Wenn es andererseits darum gehen soll, Emotionen in Experimenten zur Mensch-Roboter-Interaktion zu messen, muss die epistemische Strategie umgekehrt werden: Der mathematischen Erzeugung muss eine quantifizierende Messung zur Seite gestellt werden. Das geschieht z. B. durch Multiple-Choice-Fragebogen für menschliche Probandinnen und Probanden, die, konfrontiert mit Bildern und Videos von Robotern, ihre Gefühle einschätzen sollen (z. B. Rosenthal-von der Pütten et al. 2013). Das Facial Action Coding System (FACS) geht einen Schritt weiter, indem es Gefühle menschlicher Probanden direkt im Experiment aufzeichnet.

FACS geht auf den US-amerikanischen Psychologen Paul Ekman zurück (Ekman und Friesen 1976). Es basiert auf der Annahme, dass Mimik und Emotionen anthropologisch universell sind. Mimik sei gewissermaßen ein Gesichtsaffektprogramm, das direkter Ausdruck der Emotionen eines Menschen ist. Mittels FACS werden die Bewegungen der 98 Gesichtsmuskeln in 44 sogenannten „Action Units“ kodiert, die wiederum den Grundemotionen zugeordnet sind. Dies, so die Annahme, ermögliche den Schluss auf die hinter dem Ausdrucksverhalten liegenden Befindlichkeiten. Die Zuordnung der Gesichtsausdrücke (leicht gekräuselte Nasenwurzel, gehobene/gesenkte Mundwinkel, Augenbrauen etc.) basiert auf Datenbanken mit zehntausenden Bildern von Gesichtsausdrücken aus interkulturellen Vergleichsstudien. Es wird empfohlen, dass zwei Coder unabhängig voneinander die Videosequenzen nach diesem Schema bewerten, um ein reliables Ergebnis zu erhalten.

Die Zurechnung von Emotionen anhand des Gesichtsausdrucks ist in einer standardisierten Forschungslogik durchaus plausibel. Experimente mit FACS versuchen, subjektive Verzerrungen zu minimieren und ein komplexes Phänomen wie „Emotion“ auf überprüfbare und reproduzierbare Maße einzugrenzen. Allerdings werden die damit einhergehenden epistemischen Implikationen selten reflektiert. Neben handwerklichen Fragen zur Durchführung ${ }^{3}$ betrifft das vor allem die erkenntnistheoretische Gefahr der Ver-

\footnotetext{
${ }^{3}$ Häufig beobachtbare methodische Mängel der Durchführung betreffen das Sampling der Probanden, wo oftmals Studierende oder Mitarbeitende aus dem Zusammenhang des Labors zum
} 
wechslung des Forschungsinstruments mit dem eigentlichen Erkenntnisobjekt - den tatsächlichen Gefühlen von Menschen gegenüber Robotern. FACS erfasst nicht das Erleben einer Emotion, was für die allermeisten Menschen den wesentlichen Inhalt der Bedeutung des Wortes darstellt. Dieser Hinweis scheint vielleicht zunächst trivial. Aber genau der damit einhergehende reduktionistische Fehlschluss lässt sich im Umgang mit den Ergebnissen aus dieser Methode beobachten: Es werden Standbilder oder Videos vorgeführt, auf denen per Einblendung die annotierten Muskelgruppen farbig markiert sind. Die anhand dieser mimischer Mikrosequenzen erzeugten Werte und deren statistische Analyse werden dann in einem Graphen abgetragen, um zu belegen, dass bestimmte Begrüßungsformen dem Nutzer gefallen, ihn ängstigen etc. (Bischof 2017, S. 217-220). FACS sammelt zweifelsohne empirische Hinweise für solche Aussagen; es bleibt aber ein Instrument der Messung und nicht das eigentliche Erkenntnisobjekt. Auf das tatsächliche Gefühl und die Akzeptanz des Nutzers lässt sich von diesen laboratisierten Bedingungen - zumal ohne den Kontext der angestrebten Nutzung - nicht ohne weiteres schließen. Das vorgestellte Testsetting ist nicht der zu vermessenden Mensch-Roboter-Interaktion in realen Welten entnommen, sondern vielmehr ein Vorgehen eigener Logik. FACS ist eine technische Konfiguration eines sichtbaren Ausschnitts von Emotionen. Es ist eine wissenschaftliche Analysemethode, die in einem Laborsetting aus Kameras, Software und menschlicher Interpretation besteht. Diese Laboratisierung abstrahiert von Emotionen als Erfahrungen von Subjekten auf die regelgeleitete Hervorbringung von „Emotionen“ als an der Körperoberfläche ablesbarem Wert.

An diesem Beispiel wird deutlich, worauf Laboratisierungen als epistemische Praxis antworten: Sie sind als Reaktion auf die „complexity gap“ (Meister 2014, S. 119) zwischen alltäglichen Lebenswelten und sozialen Robotern zu verstehen. Sie sind Mittel zur Reduktion von Komplexität und Kontingenz sozialer Situationen. Solche Komplexitätsreduktionen sind (nicht nur) für Sozialrobotik notwendig. Problematischer als die notwendige Komplexitätsreduktion selbst ist die mangelnde Trennung zwischen den Instrumenten der Messung, wie FACS, und dem eigentlichen Erkenntnisobjekt, hier emotionaler Qualität in der resultierenden Mensch-Roboter-Interaktion. Der Grad der Adäquanz von Laborexperimenten mit FACS für die Mensch-Roboter-Interaktion in konkreten sozialen Situationen ist daher zunächst vollkommen unklar.

\subsubsection{Alltagsweltliche Praktiken}

Eine gelingende Mensch-Maschine-Symbiose in echten sozialen Welten wäre allein durch epistemische Praktiken der Laboratisierung also nicht adäquat erforsch- und modellierbar.

Einsatz kommen - zum Beispiel auch, wenn der avisierte Einsatz eigentlich auf die Akzeptanz des Roboters durch Menschen mit Demenz zielt. Des Weiteren wird oft von der Empfehlung abgewichen, das Material zweimal codieren zu lassen, um subjektive Verzerrungen auf der Coder-Ebene zu minimieren. 
Die Konstrukteurinnen und Konstrukteure sozialer Roboter erfahren diese Begrenzung selbst und verfolgen daher auch eine epistemische Strategie, die als Gegengewicht fungiert. Viele Forschende in der Sozialrobotik sind nämlich ausgesprochen gute Beobachterinnen und Beobachter von sozialen Welten und besitzen Erfahrungen und Fähigkeiten, die sie zur Übersetzung zwischen sozialen Situationen und sozialen Robotern einsetzen. Diese Gruppe alltagsweltlicher epistemischer Praktiken wird allerdings beinahe nie Gegenstand der offiziellen Selbstdarstellungen des Felds, was sie sehr spannend macht.

Die alltagsweltlichen epistemischen Praktiken der Sozialrobotik finden in der Regel außerhalb des Labors statt, zum Beispiel auf Universitätsfluren, bei einem „Tag der offenen Tür" oder auch im heimischen Wohnzimmer. Anders als bei Laborexperimenten werden die Forschenden hier selbst zum Instrument. Die wesentliche Ressource dieser typischen Erkenntnisform sind ihre eigenen Erfahrungen und Beobachtungen. Diese alltagsweltlichen Erkenntnispraktiken sind teilweise tief in biografischen Erlebnissen und Räsonierweisen der Forschenden selbst verankert - was sie einerseits wirksam und andererseits schwer beobachtbar macht. Wie und wieso diese für die Entwicklung von sozialen Robotern so wichtig werden, erzählen die Forschenden meist selbst, wenn man sie nach ihrem Weg in die Sozialrobotik fragt (Bischof 2017, S. 231-239; Bischof 2021).

Forschende verweisen auf eigene Erlebnisse als konstitutiv für ihr Interesse an sozialen Robotern. Zum einen handelt es sich bei diesen Erlebnissen um Ankerpunkte der eigenen Forscherbiografie, die als die weitere Entwicklung bis zum Punkt des Interviews entscheidend prägend wahrgenommen wurden. Zum anderen werden solche persönlichen Schlüsselmomente auch bei anderen Gelegenheiten wie in der Lehre oder einem Pausengespräch als beispielhafte Analogie für Mensch-Roboter-Interaktion herangezogen. Eine Postdoktorandin an einer Robotikfakultät beispielsweise beschrieb mir ihren Weg ins Feld der Sozialrobotik als vorgezeichnet durch ein Sommerpraktikum, bei dem sie ein robotisches Ausstellungsobjekt für eine Konferenz technisch vor Ort betreute. Es handelte sich um einen Roboterwurm aus Glasfaserröhrchen, der unterstützt von wechselnder Beleuchtung einen Rhythmus aus Aktivität und Ruhe vollzog. Faszinierend sei für sie dabei nicht nur die technische Arbeit an der Maschine gewesen, sondern:

I got to watch all the people coming through the conference interacting with the system without needing really explanation [...] so for me I got into robots by building them but also by seeing people interact with them. (Bischof 2017, S. 237, Hervorhebung AB)

Durch die Beobachtung von Menschen, die die Installation zum ersten Mal sahen, wurden der Forschenden die Wirkung der Installation auf Betrachtende erlebbar und somit erstmals bewusst. Die meisten Forschenden erklärten mir die besondere Eindrücklichkeit solcher Erlebnisse damit, dass die Qualität der Interaktion im Kontrast zur Ingenieursperspektive stehe (ebd., S. 235-236). Es handelt sich bei diesen Erlebnissen um die protoethnografische Beobachtung anderer Beobachter, die die Maschinen - zu denen die Forschenden oftmals eher eine alltagspraktische Hassliebe als widerständige Objekte haben - ganz anders wahrnehmen als sie selbst. Allerdings ist das eine sehr intuitive Form 
der teilnehmenden Beobachtung, die zumeist in Alltagsbegriffen und -kategorien verbleibt. Als epistemische Praktiken werden sie meist nicht schriftlich dokumentiert - obwohl sie durchaus gezielt aufgesucht und hergestellt werden können. So hat eine der prominentesten und meistzitierten Forscherinnen in der Sozialrobotik eine einfache Alltagsheuristik etabliert, um zu bewerten, ob ihre Roboter gut sind: Sie nehme jeden ihrer Prototypen für einen Nachmittag mit nach Hause, damit ihre Kinder damit spielten. An ihren Reaktionen, der Dauer und der Intensität der Beschäftigung mit der Maschine könne sie mittlerweile recht gut abschätzen, wie erfolgreich die angestrebte Mensch-RoboterInteraktion verlaufen werde (ebd., S. 248).

Diese Form von pragmatischem Alltagsexperiment ist kein Einzelfall. Ein anderer Forscher in den USA erwähnte mir gegenüber, dass er für ein laufendes Projekt ,für eine Weile Fahrstuhl gefahren“ sei (ebd., S. 244-245). Er sei dafür einen Tag lang Menschen durch das Universitätsgebäude gefolgt, „,but that wasn't really a scientific experiment“ (ebd., S. 245). Der epistemische Wert der Aktivität habe darin bestanden, den Raum bearbeitbarer Probleme einzugrenzen, um den weiteren Verlauf des Projekts zu bestimmen. Interessant ist dabei die der Methode zugeschriebene Nützlichkeit für den jetzigen Stand des Projekts. In diesem soll ein Roboter über die Universitätsflure fahren und dabei noch näher zu definierende Ausgaben ausführen. Die vom Forscher angewendete Heuristik ist dabei explorativ und typisch für alltagsweltliche epistemische Praktiken: Schauen wir einfach, wie die Leute es machen! Der protoethnografische Ausflug war also auf einen schnellen und pragmatischen Erkenntnisgewinn ausgelegt und zielte zudem auf einen bereits stark eingegrenzten Ausschnitt des beobachtbaren Verhaltens. In dieser Fokuslegung kommt auch eine das Ergebnis strukturierende These zum Ausdruck: In diesem Fall lautet sie, dass den Wegen der Menschen im Gebäude und den technischen Tasks von Robotern ähnliche Ziele zugrunde liegen.

Dennoch ist diese alltägliche Form der Beobachtung als epistemisch zu bezeichnen. Ihre Wirksamkeit geht über das Kollegengespräch hinaus, sie bestehen in einer (oftmals gezielten) Auseinandersetzung mit bestimmten Ausschnitten von Alltagswelt, um den eigenen sozialen Roboter - oder die Grundlagen seines Funktionierens - anders zu verstehen als durch ein Laborexperiment. Diese Alltagsförmigkeit verweist wiederum auf die besondere Natur des Gegenstands der Sozialrobotik, der eben nicht nur in der Komplexitätsreduktion laboratisierender Praktiken erfasst werden kann. Die alltagsweltlichen epistemischen Praktiken nehmen selbstverständlich ebenfalls Reduktionen vor. Sie finden aber in derselben Sinnprovinz wie die sozialen Gegenstände statt, was eine Integration ihrer Komplexität und Kontingenz zumindest auf der Ebene der Handlungsprobleme der Forschenden ermöglicht.

Die alltagsweltlichen Erkenntnispraktiken sind lokal, sie finden an ,echten ' Orten statt. Sie betrachten (Mensch-Roboter-)Interaktion als in einen bestimmten sozialen Kontext integriert statt isolierend. Die alltagsweltlichen Erkenntnisobjekte besitzen dabei auch eine (oft als Störung auftretende) Eigenperformanz. Sie werden nicht in formalisierten Zeichensystemen festgehalten, sondern eher mündlich mit Kolleginnen und Kollegen besprochen. Die Akteure dieser Praktiken sind die Forschenden selbst, die beobachten, inter- 
pretieren und Heuristiken verwenden. Die Beobachteten tauchen aber ebenfalls als sinnförmig Handelnde auf. Die alltagsweltlichen epistemischen Praktiken sind allerdings idiosynkratisch in dem Sinne, dass sie nicht methodisch kontrolliert werden. Ihr epistemischer Wert besteht darin, den Raum bearbeitbarer Probleme einzugrenzen, um auf eine wissenschaftliche Fragestellung hinzuarbeiten, oder sich für Designentscheidungen inspirieren zu lassen.

\subsubsection{Inszenierende Praktiken}

Forschende in der Sozialrobotik besitzen Routine darin, ihre Maschinen zu präsentieren. Die Anlässe dafür sind verschieden, stehen jedoch meist im Zusammenhang mit dem Werben für die eigene Forschung. Einen häufigen Anlass bieten professionelle Kommunikationsmaßnahmen der Institution, an der das Labor beheimatet ist. Robotikgruppen bekommen oft den Auftrag, eine Vorführung vorzubereiten, da das Interesse an Robotern allgemein hoch ist. Roboter werden auch genutzt, um potenzielle Studierende für Studiengänge zu interessieren. Wettbewerbe wie „Jugend forscht“ oder die schulischen „Science Fairs“ in den USA sind ebenfalls ein beliebtes Umfeld zur Vorführung robotischer Fähigkeiten außerhalb eines Forschungskontexts. Diesen Vorführungen kommt auch eine zentrale epistemische Qualität zu, da die Forschenden hier nicht einfach Roboterverhalten wie im Labor, mit all seinen Abbrüchen und Neustarts, oder wie in realen Nutzungssituationen, die ja nicht auf einer Bühne funktionieren, abbilden können. Sie müssen stattdessen eine Auswahl von möglichem Roboterverhalten treffen und dieses auch mit darstellerischen Mitteln wie Musik, Erzählung oder sogar eigenen Schauspieleinlagen rahmen.

Empirische Studien zeigen, dass die Interaktion mit Robotern ganz wesentlich auf der kulturellen und interaktiven Situierung durch Menschen beruht (Alač et al. 2011; Muhle 2018; Pentzold und Bischof 2019). Kein sozialer Roboter funktioniert ohne explizite Ein-

griffe und Einweisungen einer anwesenden dritten Person oder vorbereitete Weichenstellungen, bspw. durch eine Manipulation des Einsatzorts, durch Abspielen voraufgezeichneten Verhaltens oder einfach durch die populär-kulturell vermittelten Erwartungen an eine Roboterinteraktion auf Seiten der Betrachter. Erst durch diese Formen der Einbettung werden Roboter in sozialen Situationen überhaupt interaktionsfähig. Der analytische Blick auf inszenierende Praktiken in der Sozialrobotik zeigt, dass es sich dabei um eine zentrale epistemische Qualität handelt.

Die offensichtlichste Form der Inszenierungen von sozialen Robotern ist die menschenähnliche Gestaltung der Maschinen. Die allermeisten sozialen Roboter haben ein humanoides Aussehen mit Torso und Extremitäten und einem gesichtsähnlichen Fokuspunkt, der durch Ausdrucksmodalitäten wie rudimentärer Mimik oder Augenbewegungen als zentrale Schnittstelle zur Interaktion dienen soll. Hinzu kommt, dass soziale Roboter im Forschungs- und Präsentationskontext immer einen eigenen Namen erhalten und damit subjektiviert werden. Eine oftmals geäußerte Begründung dafür lautet, dass es für Menschen somit einfacher sei, mit dem Roboter zu interagieren. Empirische Untersuchungen 
zeigen dagegen, dass die durch menschenähnliche Gestaltung erzeugte Erwartung gemessen an den Fähigkeiten der Maschinen eher zu Interaktionsabbrüchen führt (Krummheuer 2010; Lindemann und Matsuzaki 2017; Muhle 2019).

Noch weiter verbreitet als eine menschenähnliche Gestaltung ist das Erstellen von Filmclips zur Demonstration von Roboterverhalten. Beinahe jedes Robotik-,,Lab“ produziert solche Videos, um die Tauglichkeit seiner Maschinen zu zeigen. Hierbei wird ein technisch bereits realisiertes oder auch erst noch zu erreichendes Roboterverhalten teils durch Fernsteuerung oder computergrafische Manipulation erzeugt und als Videoclip beispielsweise auf YouTube zirkuliert. Die Rolle solcher Clips ist sowohl für den spielerischen Ausdruck der Identität der Forschenden (Both 2015) als auch die Erzeugung von Erwartungen bei der breiteren Öffentlichkeit und Stakeholdern wie Krankenkassen (Winthereik et al. 2008) nicht zu unterschätzen. Solche Videos sind aber nicht nur persuasive Wissenschaftskommunikation, sie spielen auch für die Erkenntnispraktiken innerhalb der Robotik eine wichtige Rolle: Innerhalb einer Forschungsgruppe werden auch die Videos anderer Forschungsgruppen angeschaut und bewertet. Dabei werden die Videos auf kritische Zeichen der Inszenierung, wie Schnitte oder Beschleunigung, hin untersucht (Bischof 2017, S. 259-263). Dazu gehört auch das Wissen darüber, wie der Blick des Betrachters durch technische, filmische und symbolische Mittel gelenkt werden kann. Es besteht also nicht nur ein Wissen darüber, wie man Roboterverhalten gut inszeniert, sondern auch, wie man eine solche Inszenierung dechiffriert - als Form impliziter peer-review.

Die Beliebtheit von Videoclips sozialer Roboter zeigt sich über Demovideos hinaus in (halb-)dokumentarischen Formaten mit Roboterverhalten und Interview- oder Sprechersequenzen der Forschenden. Diese Videoclips sind ebenfalls eher kurz (typischerweise zwischen drei und fünf Minuten) und versuchen Roboterverhalten unmittelbar und affektiv darzustellen. Diese Eigenschaft wirkt besonders im Kontakt mit technischen Laien wie Forschungsförderern, Journalisten oder avisierten Nutzergruppen. Suchman (2014) hat den Charakter dieser Videos näher beschrieben: Indem Clips von Roboterfähigkeiten an übergreifende Narrative anknüpfen und technisch noch nicht mögliches Roboterverhalten simulieren, stimulieren sie ihr Publikum im Hinblick auf das Potenzial von Robotik.

Like other conventional documentary productions, these representations are framed and narrated in ways that instruct the viewer what to see. Sitting between the documentary film and the genre of the system demonstration or demo, the videos create a record that can be reliably repeated and reviewed in what becomes a form of eternal ethnographic present. These reenactments thereby imply that the capacities they record have an ongoing existence - that they are themselves robust and repeatable. (Suchman 2007, S. 237-238)

Suchman verdeutlicht hier die problematische Tendenz, dass immer wieder abrufbare Clips ein vielleicht nur einmal und kurzfristig gezeigtes Verhalten entzeitlichen. Dieses Problem besteht bei vielen Demovideos in der Robotik: Sie führen Roboterverhalten nur scheinbar neutral vor. Sie beschleunigen oder verlangsamen Bildsequenzen, sie schneiden Szenen zusammen, verstärken Wirkungen durch Musik, erklären Funktionen durch Sprechertext und schließen an Narrative und Charaktere aus Filmen und Science-Fiction- 
Büchern an. Mit diesen audiovisuellen Inszenierungen kreieren sie das Setting, vor dem die (geplante) Funktionsweise des sozialen Roboters besonders gut sichtbar werden soll.

Im Hinblick auf die dabei verwendete Rhetorik einer besseren Zukunft sind einige Analysen der symbolischen Settings von Demovideos unternommen worden. Suchman (2014) analysiert das Werbevideo für den sozialen Heimassistenten Jibo, Schulte und Graf (2020) den sozialen Heimassistenten Moxie. Die Analysen zeigen, wie sehr spezifische Ausschnitte von wünschenswertem Verhalten herangezogen werden, um die Roboter als tauglich darzustellen - und welche Aspekte sozialer Realität dabei stillschweigend nicht thematisiert werden (in den zitierten Beispielen sind es die Rollen von Klasse, Hautfarbe und Vorstellungen guter Familie).

Die Inszenierungen von Roboterverhalten in Livedemonstrationen, Demovideos oder experimentellen Anordnungen sind eine spezifische Form der Expertise, die in der Sozialrobotik weit verbreitet ist. Zur praktischen Erkenntnisweise werden sie dadurch, dass sie das Bild von sozialen Robotern und deren Fähigkeiten aktiv gestalten. Dabei werden vor allem die Eigenschaften inszeniert, die die Maschinen selbst nicht generieren können: soziale Situiertheit, symbolische Eingebundenheit, Subjektivität und Historizität. Dadurch wird das Erkenntnisobjekt Mensch-Roboter-Interaktion einerseits gestalterisch hervorgebracht, andererseits werden zukünftige Nutzungssituationen und Nutzer und Nutzerinnen beeinflusst. Die Sozialrobotikforschung schafft die Bedingungen, unter denen Mensch-Roboter-Interaktion denkbar und messbar wird, durch Praktiken der Inszenierung nicht unwesentlich selbst. Eine wichtige Funktion dieser Inszenierungen ist dabei die Erweiterung und Ermöglichung der wissenschaftlichen Fähigkeiten der Sozialrobotik. Gleichzeitig besteht die Gefahr der Mystifizierung der Maschinen in einer Umkehrung des erkenntnistheoretischen Verhältnisses dieser Forschung zu ihren Gegenständen: Sozialrobotik läuft Gefahr, ihre eigenen Inszenierungen zu erforschen, wenn die Ergebnisse der inszenierenden Praktiken nicht methodisch von den expliziten Praktiken der Wissenserzeugung, wie etwa den Laborexperimenten, getrennt werden.

\subsection{Zusammenfassung und Implikationen}

Die Erzeugung von Wissen verläuft in der Sozialrobotik anders als in naturwissenschaftlichen Disziplinen. Sie misst sich am Faktor des Gelingens einer bereits imaginierten und als nützlich bestimmten Mensch-Roboter-Interaktion statt an der Hervorbringung des Unvorhergesehenen, wie bspw. in der Biochemie (Rheinberger 2001). Die Herausforderung für den Bau sozialer Roboter ist die Passung, die Gangbarmachung der Maschinen in der sozialen Welt. Dass dabei neben Laborexperimenten auch Ressourcen wie Alltagswissen oder Inszenierung zur epistemischen Quelle werden, kann eigentlich nicht überraschen. Aber erst in ihrem Zusammenspiel werden die drei Gruppen von epistemischen Praktiken schöpferisch wirksam. Sie wechseln sich in ihren unterschiedlichen Fähigkeiten, mit sozialer Komplexität umzugehen, gewissermaßen ab. Es kommt zu einem Wechselspiel von (vorübergehendem) Ausschluss von sowie dem Streben nach (Wieder-)Eintritt von 
Komplexität und Kontingenz sozialer Welten. Die Idee für einen zu erforschenden Zusammenhang entstammt einer Alltagsbeobachtung, wird dann aber in ein isoliertes Laborszenario übertragen, um einen messbaren Effekt zu generieren. Eine explorative Nutzerstudie von Roboterverhalten, das an dieses Wissen angepasst wurde, kann wiederum den Prozess für neue Komplexitäten und Kontingenzen öffnen. Die so generierten Daten und Maschinen werden anschließend präsentiert oder auch als Video produziert (Bischof 2017, S. 265-274) (Tab. 2.1).

Der am Ende eines Sozialrobotikprojekts resultierende veröffentlichte Aufsatz ist eine sehr spezifische Form der Komplexitätsreduktion - er reduziert nicht nur die Komplexität des Einsatzes sozialer Roboter auf ein lineares, (in der Regel) erfolgreiches, quantitatives Ergebnis, sondern er macht auch viele der hier dargestellten Zwischenschritte und Einflüsse unsichtbar. Motivation und Ergebnis einer praxeologischen Laborstudie zum Bau sozialer Roboter ist daher auch, einzuschätzen, welche Implikationen sich aus diesen oftmals unsichtbaren epistemischen Praktiken für die möglichen Anwendungsfelder der Sozialrobotik ergeben. Dabei kann es natürlich nicht um die Abschätzung konkreter Akzeptanzfragen für Szenarien sozialer Roboter in Pflege, Therapie oder Sexualität gehen (z. B. Graf et al. 2020), aber um die grundlegende Konfiguration, unter der soziale Welten mit den Wissensweisen der Sozialrobotik in den Blick geraten.

Die problematischste epistemische Implikation der überwiegenden Mehrheit von Forschungszielen der Sozialrobotik (Bischof 2017, S. 174-211) ist ihre umgekehrte Forschungslogik: Sie suchen nicht ergebnisoffen nach Lösungen für Probleme in Anwendungsgebieten, sondern nach Wegen der Umsetzung des Lösungswegs „Robotereinsatz". Die Entwicklungspraxis dient zumeist dem Einsatz und der Einsetzbarkeit des Werkzeugs an sich. Meister hat auf diesen Zusammenhang schon für die Servicerobotik hingewiesen (Meister 2011, S. 120); es handelt sich erkenntnistheoretisch gesehen um eine „Post-hoc“-Ausrichtung der Forschung (Knorr Cetina 1991). Sozialrobotikprojekte, die einen partizipativen Entwicklungsprozess anstreben, also unter Beteiligung der im Anwendungsfeld tätigen Akteurinnen und Akteure, lassen sich nur selten beobachten. Obwohl solche Methoden jüngst auch im HRI-Kontext angewendet werden (Hornecker et al.

Tab. 2.1 Epistemische Praktiken der Sozialrobotik

\begin{tabular}{l|l|l|l}
\hline & Laboratisierende Praktiken & $\begin{array}{l}\text { Alltagsweltliche } \\
\text { Praktiken }\end{array}$ & $\begin{array}{l}\text { Inszenierende } \\
\text { Praktiken }\end{array}$ \\
\hline Orte & translokal & lokal & translokal \\
\hline Beobachtete Prozesse & erzeugt & selbstperformant & erzeugt \\
\hline Soziale Situiertheit & isoliert & integriert & erzeugt \\
\hline Epistemische Akteure & $\begin{array}{l}\text { soziotechnische } \\
\text { Aufzeichnungssysteme }\end{array}$ & Alltagsmenschen & ,belebte' Roboter \\
\hline Zeichensysteme & formalisiert & idiosynkratisch & kulturell geteilt \\
\hline $\begin{array}{l}\text { Selbstverständnis } \\
\text { Forschende }\end{array}$ & passiv & aktiv & expressiv \\
\hline
\end{tabular}

Epistemische Praktiken der Sozialrobotik in der Übersicht (eigene Darstellung) 
2020; Björling und Rose 2019; Lee et al. 2017; Lee und Riek 2018), schützt die Verwendung solcher Methoden allein nicht vor einer Dekontextualisierung der sozialen Nutzungssituation. Auch partizipative Methoden konfigurieren zukünftige Nutzer in spezifischer Weise (Bischof und Jarke 2001), je nachdem, wann diese in ein Projekt eingebunden werden und wie einflussreich deren Urteil für die Umsetzung von Änderungen am Prototyp oder Projektziel ist. Das Versprechen der Nützlichkeit als wesentlicher epistemischer Bedingung der Konstruktion sozialer Roboter kann sich nur realisieren, wenn die Entwicklungspraxis tatsächlich an der Adäquanz der Mensch-Roboter-Interaktion in Alltagswelten ausgerichtet ist.

Die zweite Implikation betrifft die Sichtbarkeit, Nachvollziehbarkeit und damit Möglichkeit der methodischen Kontrolle der epistemischen Praktiken der Sozialrobotik selbst. Die sehr hilfreichen alltagsförmigen Praktiken und auch die eigene Rolle in den Inszenierungen werden weder dokumentiert noch methodisch reflektiert. Die Reflexion methodischer Implikationen auch dieser nicht unter das wissenschaftliche Selbstbild fallender - aber sehr wohl elementarer - epistemischer Praktiken wäre wiederum die Voraussetzung für eine einbindende und aufsuchende Einbindung von sozialen Welten in den Entwicklungsprozess. Ein partizipativer Designprozess basiert ganz wesentlich auf solchen alltagsförmigen epistemischen Praktiken - und ihrer methodischen Kontrolle (Bischof 2021). Aus Angst vor blinden Flecken und subjektiven Verzerrungen die Bedeutung alltagsweltlicher oder inszenierender Praktiken für die Produktion von Wissen über Mensch-Roboter-Interaktion in der wissenschaftlichen Selbstdarstellung auszublenden, verhindert gerade ihre Dokumentation und Analyse. Der Bau erfolgreicher sozialer Roboter wird immer auf Wissen und Praktiken aus diesen unterschiedlichen Sinnprovinzen angewiesen sein. Adäquate Mensch-Roboter-Interaktion lässt sich im Labor allein nicht erzeugen.

\section{Literatur}

Alač M, Movellan J, Tanaka F (2011) When a robot is social: spatial arrangements and multimodal semiotic engagement in the practice of social robotics. Soc Stud Sci 41(6):893-926

Asimov I (2014) I, Robot. Spectra, New York

Becker-Asano C, Wachsmuth I (2008) Affect simulation with primary and secondary emotions. Intelligent virtual agents. Springer, Berlin/Heidelberg

Bendel O (Hrsg) (2018) Pflegeroboter. Springer Gabler, Wiesbaden

Bischof A, Jarke J (2001) Configuring the older adult: How age and ageing are re-configured in gerontechnology design. In: Peine A, Marshall B, Martin W, Neves L (Hrsg) Socio-gerontechnology. Interdisciplinary Critical Studies of Ageing and Technology. Routledge, London, S 197-212

Bischof A (2015) Wie Laborexperimente die Robotik erobert haben. Einblick in die epistemische Kultur der Sozialrobotik. In: Maibaum A, Engelschalt J (Hrsg) Auf der Suche nach den Tatsachen: Proceedings der 1. Tagung des Nachwuchsnetzwerks „INSIST“, Berlin, S 113-126

Bischof A (2017) Soziale Maschinen bauen. Epistemische Praktiken der Sozialrobotik. transcript, Bielefeld 
Bischof A (2020) „Wir wollten halt etwas mit Robotern in Care machen“. Epistemische Bedingungen der Entwicklungen von Robotern für die Pflege. In: Hergesell J, Maibaum A, Meister M (Hrsg) Genese und Folgen der Pflegerobotik. Beltz-Juventa, Weinheim, S 46-61

Bischof A (2021) Körper, Leib und Mystifizierung in der Gestaltung von Mensch-RoboterInteraktion. In: Escher C, Tessa Zahner N (Hrsg) Begegnung mit dem Materiellen. Erfahrung mit Materialität in Architektur und Kunst. transcript, Bielefeld, S 213-228

Bischof A, Maibaum A (2020) Robots and the complexity of everyday worlds. In: Goecke BP, Rosenthal-von der Pütten A (Hrsg) Artificial intelligence. Reflections in philosophy, theology, and the social sciences. Mentis, Paderborn, S 307-320

Björling E, Rose E (2019) Participatory research principles in human-centered design: engaging teens in the co-design of a social robot. MTI 3(1):8

Böhle K, Bopp K (2014) What a vision: the artificial companion. A piece of vision assessment including an expert survey. Sci Technol Innov Stud 10(1):155-186

Both G (2015) Youtubization of research. Enacting the high tech cowboy through video demonstrations. Stud Sci Commun 24:24-53

Breazeal C (2003) Emotion and sociable humanoid robots. Int J Human Comput Stud 59:119-155

Brooks R (1999) Cambrian intelligence: the early history of the new AI. MIT Press, Cambridge, MA

Čapek K (2014) R.U.R. Rossum's universal robots. A play in introductory scene and three acts. eBooks, Adelaide

Clarke R (2011) Asimov's laws of robotics, implications for information technology. In: Anderson M, Anderson SL (Hrsg) Machine ethics. Cambridge University Press, Cambridge, S 254-285

Ekman P, Friesen W (1976) Measuring facial movement. Environ Psychol Nonverbal Behav 1(1):56-75

Engelberger J (2012) Robotics in practice: management and applications of industrial robots. Springer Science \& Business Media, Berlin/Heidelberg

Graf P, Maibaum A, Compagna D (2020) Care, therapy, or sex robots? TATuP - Z Technikfolgenabschätzung in Theorie und Praxis 29(2):52-57

Hergesell J, Maibaum A, Meister M (2020) Genese und Folgen der Pflegerobotik. Die Konstitution eines interdisziplinären Forschungsfeldes. Beltz-Juventa, Weinheim

Hornecker E, Bischof A, Graf P, Franzkowiak L, Krüger N (2020) The interactive enactment of care technologies and its implications for human-robot-interaction in care. In: Proceedings NordiCHI 2020. ACM Tallinn, 1-11

Jasanoff S, Kim S (2009) Containing the atom: sociotechnical imaginaries and nuclear power in the United States and South Korea. Minerva 47(2):119-146

Knorr Cetina K (1988) Das naturwissenschaftliche Labor als Ort der „Verdichtung“ von Gesellschaft. Z Soziol 17:85-101

Knorr Cetina K (1991) Epistemic cultures: forms of reason in science. Hist Polit Econ 23(1):105-122

Krey B (2014) Michael Lynch: Touching paper (s) - oder die Kunstfertigkeit naturwissenschaftlichen Arbeitens. In: Lengersdorf D, Wieser M (Hrsg) Schlüsselwerke der Science \& Technology Studies. Springer Fachmedien, Wiesbaden, S 171-180

Krummheuer A (2010) Interaktion mit virtuellen Agenten. Zur Aneignung eines ungewohnten Artefakts. Lucius, Stuttgart

Kuhn T (1957) The Copernican revolution: planetary astronomy in the development of western thought. Harvard University Press, Cambridge, MA

Lee HR, Riek L (2018) Reframing assistive robots to promote successful aging. ACM Trans Hum Robot Interact 7(1):1-23 
Lee HR, Šabanović S, Chang W-L, Nagata S, Piatt J, Bennett C, Hakken D (2017) Steps toward participatory design of social robots. In: HRI'17. Proceedings of the ACM/IEEE international conference on human-robot interaction: March 6-9, 2017, S 244-253

Lindemann G (2016) Social interaction with robots: three questions. AI \& Soc 31(4):573-575

Lindemann G, Matsuzaki H (2017) Die Entwicklung von Servicerobotern und humanoiden Robotern im Kulturvergleich - Europa und Japan. In: DFG-Abschlussbericht 2017. https://www.unioldenburg.de/fileadmin/user_upload/sowi/ag/ast/DFG-Projekt_LI_9763-1_Abschlussbericht_2017.pdf. Zugegriffen am 14.03.2021

Lipp B (2019) Interfacing RobotCare. Doctoral dissertation, Technische Universität München, München. https://mediatum.ub.tum.de/doc/1472757/file.pdf. Zugegriffen am 14.03.2021

Lipp B (2020) Genealogie der RoboterPflege. Zur politischen Rationalität des europäischen Innovationsdispositivs. In: Hergesell J, Maibaum A, Meister M (Hrsg) Genese und Folgen der Pflegerobotik. Beltz-Juventa, Weinheim, S 18-45

Maibaum A, Bischof A, Hergesell J, Lipp B (2021) A critique of robotics in health care. AI \& Society. doi:10.1007/s00146-021-01206-Z

Meinecke L, Voss L (2018), I Robot, you unemployed': science-fiction and robotics in the media. In: Engelschalt J, Maibaum A, Engels F, Odenwald J (Hrsg) Schafft Wissen: Gemeinsames und geteiltes Wissen in Wissenschaft und Technik: Proceedings der 2. Tagung des Nachwuchsnetzwerks „INSIST“, Karlsruhe, S 203-221

Meister M (2011) Soziale Koordination durch Boundary Objects am Beispiel des heterogenen Feldes der Servicerobotik. Dissertation, Fakultät Planen, Bauen, Umwelt. Technische Universität Berlin, Berlin

Meister M (2014) When is a robot really social? An outline of the robot sociologicus. Sci Technol Innov Stud 10(1):107-134

Muhle F (2018) Sozialität von und mit Robotern? Drei soziologische Antworten und eine kommunikationstheoretische Alternative. Z Soziol 47(3):147-163

Muhle F (2019) Humanoide Roboter als ,technische Adressen“: Zur Rekonstruktion einer MenschRoboter-Begegnung im Museum. Sozialer Sinn 20(1):85-128

Mutlu B, Forlizzi J (2008) Robots in organizations: the role of workflow, social, and environmental factors in human-robot interaction. In: 2008 3rd ACM/IEEE international conference on humanrobot interaction, Amsterdam, S 287-294

Nourbakhsh I (2013) Robot futures. MIT Press, Cambridge, MA

Parviainen J, Coeckelbergh, M (2020) The political choreography of the Sophia robot: beyond robot rights and citizenship to political performances for the social robotics market. AI \& Society. doi:10.1007/s00146-020-01104-w

Pentzold C, Bischof A (2019) Making affordances real: socio-material prefiguration, performed agency, and coordinated activities in human - robot communication. Soc Media Soc 5(3):2056305119865472

Rheinberger H (2001) Experimentalsysteme und epistemische Dinge: eine Geschichte der Proteinsynthese im Reagenzglas. Wallstein, Göttingen

Rittel H, Webber M (1973) Dilemmas in a general theory of planning. Policy Sci 4(2):155-169

Rosenthal-von der Pütten A, Krämer N, Hoffmann L, Sobieraj S, Eimler S (2013) An experimental study on emotional reactions towards a robot. Int J Soc Robot 5(1):17-34

Šabanović S (2007) Imagine all the robots: developing a critical practice of cultural and disciplinary traversals in social robotics. Doctoral Thesis Faculty of Rensselaer Polytechnic Institute

Šabanović S (2010) Robots in society, society in robots mutual shaping of society and technology as a framework for social robot design. Int J Soc Robot 2(4):439-450

Schulte B, Graf P (2020) Child care robot: moxie. an analysis of the companion robot moxie. Blog Post. http://www.rethicare.info/publications/moxie/. Zugegriffen am 14.03.2021 
Suchman L (2007) Human-machine reconfigurations: plans and situated actions. Cambridge University Press, Cambridge, MA

Suchman L (2014) Humanizing humanity. Blog Post. https://robotfutures.wordpress.com/2014/07/19/ humanizing-humanity/. Zugegriffen am 14.03.2021

Treusch P (2015) Robotic companionship: the making of anthropomatic kitchen robots in queer feminist technoscience perspective. Doctoral dissertation. Linköping University Electronic Press, Linköping. http://urn.kb.se/resolve?urn=urn\%3Anbn\%3Ase\%3Aliu\%3Adiva-118117. Zugegriffen am 14.03.2021

Voss L (2021) More than machines?: The attribution of (in) animacy to robot technology. transcript, Bielefeld

Wagner C (2013) Robotopia Nipponica. Recherchen zur Akzeptanz von Robotern in Japan. Tectum, Marburg

Winthereik B, Johannsen N, Strand D (2008) Making technology public. Inf Technol People 21:116-132

Open Access Dieses Kapitel wird unter der Creative Commons Namensnennung 4.0 International Lizenz (http://creativecommons.org/licenses/by/4.0/deed.de) veröffentlicht, welche die Nutzung, Vervielfältigung, Bearbeitung, Verbreitung und Wiedergabe in jeglichem Medium und Format erlaubt, sofern Sie den/die ursprünglichen Autor(en) und die Quelle ordnungsgemäß nennen, einen Link zur Creative Commons Lizenz beifügen und angeben, ob Änderungen vorgenommen wurden.

Die in diesem Kapitel enthaltenen Bilder und sonstiges Drittmaterial unterliegen ebenfalls der genannten Creative Commons Lizenz, sofern sich aus der Abbildungslegende nichts anderes ergibt. Sofern das betreffende Material nicht unter der genannten Creative Commons Lizenz steht und die betreffende Handlung nicht nach gesetzlichen Vorschriften erlaubt ist, ist für die oben aufgeführten Weiterverwendungen des Materials die Einwilligung des jeweiligen Rechteinhabers einzuholen.

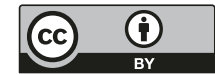

